Conclusions Direct adherent and modicum medium changing method is the best one for MSC isolation and culture. $11 \%$ is the most suitable serum concentration for MSC growth.

\section{e0174 EFFECT OF HIF1A ON PROLIFERATION AND DIFFERENTIATION OF MSC UNDER HYPOXIA CONDITION IN VITRO}

doi:10.1136/hrt.2010.208967.174

${ }^{1}$ Li Xueyuan, ${ }^{2}$ Zhang Yang. ${ }^{1}$ The First Hospital of China Medical University, Shenyang, Liaoning, China; ${ }^{2}$ The Centrol Affiliated Hospital of Shenyang Medical College, Shenyang, Liaoning, China

Aims To investigate the effect of HIF-1a on MSC under hypoxia condition.

Materials and methods We transfected HIF-1a into MSC of P3 generation through liposome 2000, and observed the expression of green fluorescence protein in order to assess transfecting efficiency. G418 was used to screen stable transfected cells, and limited dilution method used for monoclone culture of screened cells. We identified the stable HIF-1a transfected MSC through the cell surface antigen testing. We compared the growth state among stable transfected MSC with HIF-1a, vacant plasmid transfected MSC and untransfected MSC under hypoxia condition, and the expression of HIF-1a mRNA, VEGF mRNA, HIF-1a protein and VEGF protein was tested. Results pcDNA3.0-HIF-1a-eGFP can be successfully transfected into MSC mediated by liposome 2000, with efficiency of $21 \%$. Stable monoclone of transfected MSC can be obtained by G418 screening and limited dilution method. Stable transfected MSCs still reserve the ability of differentiating to chondrocyte and lipocyte. MSCs transfected with pcDNA3.0-HIF-1a-eGFP had lower apoptosis $(p<0.05)$, greater proliferation $(p<0.05)$, and more expression of HIF-1a mRNA, VEGF mRNA, HIF-1a protein, VEGF protein than MSCs transfected with vacant plasmid pcDNA3.0- eGFP and untransfected ones under hypoxia condition.

Conclusions Stable transfected MSC with HIF-1a has a significant high expression of HIF-1a protein, HIF-1a mRNA, VEGF protein and VEGF mRNA under hypoxia condition. HIF-1a could reduce MSC apoptosis and enhance its proliferation under hypoxia condition.

\section{e0175 THE EFFECT OF GHRELIN ON THE REGRESSION OF ATHEROSCLEROSIS PLAQUE IN APOE-/- MICE AORTA}

doi:10.1136/hrt.2010.208967.175

Deng Bin, Xie Xiumei, Fang Li, Chen Xiaobin, Chen Meifang. Department of Cardiology, Xiangya Hospital, Central South University, Changsha, Hunan, China

Objective To observe the effect of ghrelin on reducing the $\mathrm{apoE}^{-/-}$ mice plasma IL-8, MCP-1, TNF $\alpha$ level and the NFKBp65 expression in vascular wall and the regression of atherosclerotic plaque.

Method 8 week $\mathrm{ApoE}^{-/-}$mice were fed with Western style meals, and the same age mice C57BL/6J fed with the same meals as control. In the eighth week, $\mathrm{ApoE}^{-/-}$mice were assigned to ghrelin intraperitoneal injection and saline injection group randomly in the twelfth week.All of the groups had blood drawn from eye sockets, with isolated plasma used to measure IL-8, MCP-1, TNF $\alpha$ by ELISA. Mice were killed for examination with stereomicroscopy and paraffin imbedding for $\mathrm{HE}$ and immunohistochemistry, and frozen section for red oil stain.

Result 1. On stereomicroscopy, HE, oil red stain and image analysis equipment measurement demonstrated no plaque at C57BL/6J mice vessels, and both apoE ${ }^{-/-}$group and $\mathrm{ApoE}^{-/-}+$ghrelin groups had atherosclerosis plaque at vessels $(22.56 \pm 2.2$ vs $32.37 \pm 3.2 \mathrm{p}<0.01)$ 2. Contrast to $\mathrm{C} 57 \mathrm{BL} / 6 \mathrm{~J}$ mice, apoE ${ }^{-1-}$ mice has higher plasma TNF $\boldsymbol{\alpha}$, IL-8, MCP-1level $(28.81 \pm 1.8$ vs $11.5 \pm 0.6, \mathrm{p}<0.05 ; 335 \pm 16.7$ vs
$25.0 \pm 2.0, \mathrm{p}<0.05 ; 78 \pm 5.6$ vs $15.8 \pm 2.0, \mathrm{p}<0.05)$, but $\mathrm{apoE}^{-/}+$ghrelin mice has lower TNF $\alpha$, IL-8, MCP-1 level than $\mathrm{ApoE}^{-1-}$ mice $(15.45 \pm 0.98$ vs $24.5 \pm 1.68, p<0.05 ; 168.32 \pm 8.78$ vs $335 \pm 16.7 p<0.05$; $45.5 \pm 4.5$ vs $78.5 \pm 5.6, \mathrm{p}<0.05)$. 3. Contrast to C57BL/6J mice, apoE ${ }^{-1}$ mice NFKBp65 immunohistochemistry positive cell integral calculus value were increase $(1424.23 \pm 167.80$ vs $6859.68 \pm 675.34, p<0.01)$; ghrelin $+\mathrm{apoE}^{-/-}$mice NFKBp65 immunohistochemistry positive cell integral calculus value was lower than $\mathrm{apoE}^{-/-}$mice (3424.78 \pm 321.6 vs 6859.68 $\pm 675.34, p<0.01)$, ghrelin can decrease the expression of $\mathrm{NF \kappa Bp} 65$ in apoE $\mathrm{E}^{-1-}$ mice aorta.

Conclusion Ghrelin can inhibit the inflammatory response to decrease $\mathrm{ApoE}^{-1-}$ mice atherosclerosis plaque formation.

\section{e0176 THE EFFECTS OF ROSUVASTATIN ON THE EXPRESSION OF HOMOCYSTEINE-INDUCED EXPRESSION OF MATRIX METALLOPROTEINASE-2 (MMP-2) AND CELL MIGRATION IN RAT VASCULAR SMOOTH MUSCLE CELLS}

doi:10.1136/hrt.2010.208967.176

Yangbo Xing, Hangyuan Guo, Yafei Shi. Department of Cardiology, Shaoxing People Hospital, Shaoxing, China

Objective The aim of this study was to investigate the effects of rosuvastatin on the expression of homocysteine-induced expression of matrix metalloproteinase-2 (MMP-2) and cell migration in rat vascular smooth muscle cells (VSMC).

Methods Cultured rat VSMC were incubated with different concentrations of Hcy and rosuvastatin (Hcy $1000 \mu \mathrm{mol} / \mathrm{l}$ ) in vitro for 24, 48 and $72 \mathrm{~h}$. The expression of MMP-2 was determined by using the methods of gelatin zymography and western blotting. Cultured rat VSMC was incubated with different concentrations of Hcy and rosuvastatin (Hcy $1000 \mu \mathrm{mol} / \mathrm{l}$ ) in transwell for 24, 48 and $72 \mathrm{~h}$. The number of VSMC which transited the membrane represented the aggressivity of VSMC.

Results Hcy $(50 \sim 1000 \mu \mathrm{mol} / \mathrm{l})$ increased the expression and activity of MMP-2 significantly. Incubated with the same concentration of Hcy the expression and activity of MMP- 2 of $72 \mathrm{~h}$ was higher than that of $24 \mathrm{~h}$ and $48 \mathrm{~h}$. Hcy reduced the expression of MMP-2 at the concentration of $5000 \mu \mathrm{mol} / \mathrm{l}$. Rosuvastatin could inhibit the augmentation of homocysteine-induced expression and activity of MMP-2. Hcy $(50 \sim 5000 \mu \mathrm{mol} / \mathrm{l})$ could stimulate the migration of VSMC. Rosuvastatin could decrease the stimulation of homocysteine-induced migration of VSMC.

Conclusions These data suggested that Hcy can increase the MMP-2 expression/activity and the migration of VSMC. It may be one of the roles in the pathogenesis of atherosclerosis induced by Hcy. Rosuvastatin can inhibit the augmentation of homocysteineinduced MMP-2 expression/activity and migration of VSMC. This may be one of the pleiotropic of rosuvastatin besides lipid-lowering and benefit the therapy of CHD.

\section{e0177 EXPLORATION NEW METHODS FOR ESTABLISHMENT OF PORCINE MODEL OF ACUTE MYOCARDIAL INFARCTION}

doi:10.1136/hrt.2010.208967.177

Siming Tao, Tao Guo, Shunhua Pu, Zhuo Yu. The First Affiliated Hospital of Kunming Medical College, Kunming, China

Objective To explore and develop one optimise method that it could establish the porcine model of acute myocardial infraction more safer, quicker, convenient than routine methods.

Methods 30 animals with health condition, mean weight $26.5 \pm 4.8 \mathrm{~kg}$; The pigs were divided into two groups randomly, group A ( $\mathrm{n}=13)$ and group $\mathrm{B}(\mathrm{n}=17)$, according to different method. Angioplasty balloon was positioned in the mid-distal of left anterior 